\title{
Twin Studies in Medical and Clinical Genetics
}

\section{TWIN STUDIES IN MEDICAL GENETIGS}

\author{
P. PROPPING, F. VOGEL \\ Institute of Anthropology and Human Genetics, University \\ of Heidelberg, German Federal Republic
}

Abstract not received

\section{TWIN STUDIES \\ IN GLINIGAL GENETICS \\ (THE CLINICAL COTWIN METHOD)}

\section{GEDDA}

The Gregor Mendel Institute of Medical Genetics and Twin Research, Rome, Italy

The purpose of this review is to bring out the value of one couple from a clinical point of view, that is, when disease strikes a pair of twins. We gave this type of study the name of "Clinical Cotwin Method". The CGTM differs from the Cotwin Method because it also concerns the $\mathrm{DZ}$ twins and because it is not an experimental program but is produced by the spontaneous presence of the disease.

In the case of concordant disease in $\mathrm{MZ}$ twins, the points of interest suggested by the CCTM are the following:

I. Study of etiopathogenesis of the disease.

2. Study of the genetic variability in the manifestation of such disease.

3. Study of the variability of expression of the morbid genotype.

4. Study of the chronogenetic variability, that is, the possible difference between times of onset of disease, of its symptoms, and of its outcome.

In the case of discordant disease in $\mathrm{MZ}$ twins, two eventualities could happen, that is, one twin could be ill and the other healthy, or else, both twins could have two different diseases.

In the first case, the clinician finds an excellent basis of comparison in the healthy twin, which allows him to extend his knowledge of the morbid phenotype as well as to discover minimal symptoms which would go unobserved but for the possibility of such a detailed and individualized comparison.

In the case of two different diseases which strike MZ twins simultaneously, comparative analysis of the general symptoms is possible; for example, the variability of fever, blood chemistry, red and white blood cells, neuromuscular reaction, etc., with respect to different diseases. In this case, the CCTM allows a deeper knowledge of the etiopathogenesis of the morbid processes.

As concerns $\mathrm{DZ}$ twins, even though they do not offer the exceptional advantage of a "coeteris paribus" point of departure, they too represent a matter of high scientific interest because their semiequivalent genotypes have simultaneous times of departure. This situation differs from that of full sibs and that of half sibs; it is also very useful to analyze the disease that $\mathrm{DZ}$ twins face in the same physical time.

On the basis of the Mendel Institute's twenty years of experience, it can be said that the treatment of twins and scientific research can be greatly helped by hospital departments or institutes specializing in treatment of $\mathrm{MZ}$ and $\mathrm{DZ}$ twins, as well as outpatient departments or consultants for occasional and routine visits, both generic and specialized.

Prof. Luigi Gedda, Istituto Mendel, Piazza Galeno 5, oo161 Roma, Italy 